\title{
Quantum interferometer combining squeezing and parametric amplification
}

\author{
Xiaojie Zuo ${ }^{1}$, Zhihui Yan ${ }^{1,2}$ Yanni Feng ${ }^{1}$, Jingxu $\mathrm{Ma}^{1}$, Xiaojun $\mathrm{Jia}^{1,2}{ }^{\mathrm{O}}$ Changde Xie ${ }^{1,2}$, and Kunchi Peng ${ }^{1,2}$ \\ ${ }^{1}$ State Key Laboratory of Quantum Optics and Quantum Optics Devices, \\ Institute of Opto-Electronics, Shanxi University, Taiyuan, 030006, P. R. China \\ ${ }^{2}$ Collaborative Innovation Center of Extreme Optics, Shanxi University, Taiyuan 030006, P. R. China
}

\begin{abstract}
High precision interferometers are the building blocks of precision metrology and the ultimate interferometric sensitivity is limited by the quantum noise. Here we propose and experimentally demonstrate a compact quantum interferometer involving two optical parametric amplifiers and the squeezed states generated within the interferometer are directly used for the phase-sensing quantum state. By both squeezing shot noise and amplifying phase-sensing intensity the sensitivity improvement of $4.86 \pm 0.24 \mathrm{~dB}$ beyond the standard quantum limit is deterministically realized and a minimum detectable phase smaller than that of all present interferometers under the same phase-sensing intensity is achieved. This interferometric system has significantly potential applications in a variety of measurements for tiny variances of physical quantities.
\end{abstract}

Metrology underpins the quantitative science and the improvement of measurement precision leads to not only extensive detailed knowledge but also new fundamental understanding of nature. The classical interferometer consisting of linear beam splitters for optical beam splitting and recombination is a powerful metrology tool and the phase change of light in the interferometer is quite sensitive to a variety of variances of physical quantities influencing the optical path, such as biological samples [1], continuous force and displacement [2]. Recently, the gravitational-wave signals from the mergers of two binary black holes and neutron stars have been observed by kilometer-scale laser interferometers [3]. However, the sensitivity for current interferometer is limited by the vacuum fluctuations of electromagnetic field inside the interferometer, which is generally called the shot noise limit (SNL): $\Delta \phi_{S N L}=1 / \sqrt{N}$ [4, 5]. The SNL is the limitation of the precision for a classical optical device because the existence of shot noise impedes its further improvement.

Quantum metrology employs quantum resources to improve the measurement precision for breakthrough of the classical precision limit [6, 7]. In quest for high precision measurement, it has been demonstrated that the sensitivity determined by classically behaving states can be surpassed if exotic quantum states are applied [8]. A photon number maximally entangled state (NOON state) has been applied in interferometer measurements in which the phase signals have been enhanced by $\mathrm{N}$ times and the sensitivities have been increased beyond the classical limit [9-11]. In the Bose-Einstein condensates, the classical limit has been beaten by using the entangled states to cancel quantum noise via quantum destructive interference [12, 13]. The vacuum fluctuations have been significantly suppressed by making the use of the squeezed states and the sensitivities beyond the SNL have been achieved [14, 15]. The squeezed state injection into the interferometric gravitational-wave detectors to further improving sensitivities is progressing [16-19]. In quantum mechanics, the Heisenberg uncertainty gives the ultimate limit of sensitivity, which is named as the Heisenberg limit (HL): $\Delta \phi_{H L}=1 / N$ [20]

\footnotetext{
*zhyan@sxu.edu.cn

$\dagger$ jiaxj@sxu.edu.cn
}

and lots of efforts have been made to pursue the HL [21-26]. Especially, it is possible to reach the HL by driving the interferometers with the squeezed states in principle [27, 28].

On the other hand, the interferometers with novel structures provide an alternative avenue to achieve high precision phase sensing. The parametric processing has been wildly adopted in construction of interferometers for realizing quantum metrology [29-31]. The four wave mixing (FWM) instead of linear beam splitters have been used for optical beam splitting and recombination to form a SU(1,1) interferometer with an improvement in sensitivity, where the signal related to the phase change is enhanced while the noise level is kept close to the SNL [32, 33]. Recently, based on utilizing the truncated $\mathrm{SU}(1,1)$ interferometers several groups have also demonstrated the enhancement of sensitivity by the amplification of the signal and the reduction of the quantum noise [3436] and implemented the quantum-enhanced measurement of microscopic cantilever displacement [37, 38]. In the Ref [34], the two-mode squeezed state generated by FWM, is used as probe of interferometer. The second nonlinear interaction in the $\mathrm{SU}(1,1)$ is replaced with two balanced homodyne detections (BHDs), which is used to the direct measurement of the phase-sensing fields. The joint quadratures improve the phase sensitivity in the truncated SU(1,1) interferometers by both amplifying the phase-sensing intensity and squeezing the shot noise below the SNL. In the truncated SU(1,1) interferometer the phase-sensing field is directly injected into the detectors, thus its intensity has to be limited below the saturation power of detectors. In the Ref [39], an optical parametric amplifier (OPA) is inserted into the single-photon-based interferometer in the presence of losses and the achievable interferometric sensitivity based on the heralded single-photon probe is improved, and still scales as $\sqrt{\mathbf{N}}$. As well-known, the OPA consisting of an optical cavity with a $\chi^{(2)}$ nonlinear crystal is a stable solid quantum device to reduce shot noise of optical fields [40-47], with which the highest squeezing of 15 $\mathrm{dB}$ to date is achieved [48]. Due to both favorable features of noise squeezing and signal amplification the OPA should be a good quantum optical resource to be applied for constructing a quantum interferometer with the sensitivity beyond the SNL. So far, the deterministically experimental realization of phase sensing with high precision is still a significant challenge in quantum metrology. 
In this letter we propose and demonstrate a feasible approach to construct a quantum interferometer by combining squeezing and parametric amplification. For interferometric metrology, the phase-sensing intensity is associated with the interferometric sensitivity and the higher phase-sensing intensity allows the better interferometric sensitivity. However, the ultimate limitation of sensitivity is quantum noise of the phase-sensing light. Thus, to implement a precise interferometric measurement the phase-sensing light with higher intensity and as low as possible noise is wanted. For achieving both squeezing of shot noise and amplifying of phase-sensing intensity within a Mach-Zehender (MZ) interferometer, two OPAs are placed in two arms of the interferometer, respectively. The squeezed states generated within the interferometer are utilized as the phase-sensing quantum states. Due to effectively exploiting shot noise squeezing and parameter amplifying features of OPAs, the sensitivity of the interferometer is deterministically improved, and the sub-SNL scaling sensitivity is achieved. The experimental results show that the squeezed noise floor of the output signal optical beam from the interferometer is $5.57 \pm 0.19 \mathrm{~dB}$ below the SNL when the phase-sensing intensity is amplified from $5 \mu \mathrm{W}$ to $75.3 \mu \mathrm{W}$. An enhancement of $4.86 \pm 0.24 \mathrm{~dB}$ in the signal to noise ratio (SNR) in comparison with the classical device are measured. When the phase-sensing intensity is $75.3 \mu \mathrm{W}$, the calculated shot noise spectral density is $6.20 \times 10^{-8} / \sqrt{\mathrm{Hz}}$. Our measurement results have reached the Heisenberg-scale precision under low phase-sensing intensity. Using the presented system, only by simply manipulating the OPA gain the optimal phase sensitivity can be achieved. In the presented OPA-based quantum interferometer the squeezed state of light generated by OPA inside the interferometer is used as the phase-sensing probe and directly interacted with the measured sample, so the transmission losses is significantly reduced. In our interferometer the destructive interference output of phase-sensing fields is selected as the signal fields measured by BHDs. In this case, the measured intensities are low enough, thus the problem of power saturation for detectors is overcome. Without the power limitation to signal fields of BHDs the presented system can be used not only for the measurement of microscopic phase-sensing intensity, but also for that of higher phase-sensing intensity.

Fig. 1 (a) is schematic diagram of a MZ interferometer involving two OPAs. At first, the coherent laser $\hat{a}_{i n}$ together with the vacuum state $\hat{b}_{i n}$ are injected into the interferometer and splitted into two modes $\hat{A}$ and $\hat{B}$ by the linear 50:50 beam splitter $\mathrm{BS}_{1}$. Then, $\hat{A}$ and $\hat{B}$ are amplified by two OPAs to be $\hat{C}$ and $\hat{D}$, respectively. The mode $\hat{D}$ passes through a sample to be measured, which will give rise to a phase change $\delta$ of $\hat{D}$. Next, the two beams $\hat{C}$ and $\hat{D}$ are recombined by the linear 50:50 beam splitter $\mathrm{BS}_{2}$ to produce the output modes $\hat{a}_{\text {out }}$ and $\hat{b}_{\text {out }}$. The resultant interference signal $\hat{b}_{\text {out }}$ is sensitive to the phase change $\delta$. Instead of measuring photon intensity, we measure the quadrature phase $\hat{P}$ of $\hat{b}_{\text {out }}$ to obtain the signal related to phase change $\delta$, which is implemented by means of BHD. For a direct measurement processing the electronics noise will swamp the weak signal, which is called (a)
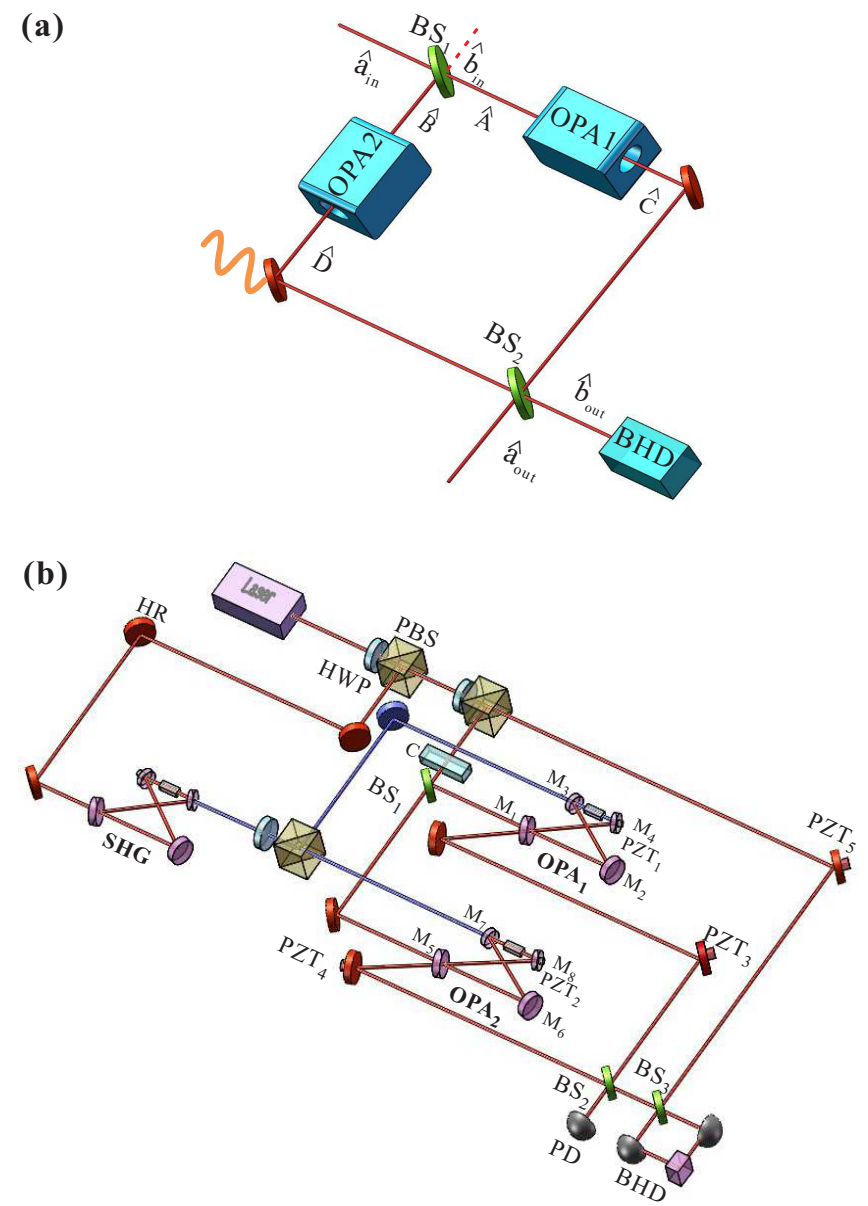

FIG. 1. (a) Schematic diagram for the quantum interferometer. The output fields from OPAs are utilized as the phase-sensing light and the BHD measures the quadrature phase of output optical field related to the phase change $\delta$. (b) Experimental setup for implementing the phase change measurement using Mach-Zehender interferometer with two OPAs. The phase change $\delta$ is mimicked by the $\mathrm{PZT}_{4}$. SHG: Second harmonic generation. OPA 1,2 : Optical parametric amplifier. BHD: Balanced homodyne detection. $\mathrm{BS}_{1-3}$ : 50:50 beam splitter. HR: high-reflection mirror. $\mathrm{M}_{1-8}$ : cavity mirror. HWP: Half-wave plate. PBS: Polarization beam splitter. $\mathrm{PZT}_{1-4}$ : piezoelectric-transducer. C: Chopper with attenuator.

as the dark count problem. In the BHD, a strong local oscillator is employed to amplify the quadrature components of weak sideband modes of a signal field. In this case the dark count problem is overcome. While the relative phase between the optical paths of two arms in the interferometer is kept to be $\pi+2 k \pi$ ( $k$ is an integer) to obtain the destructive interference, the quadrature phase $\hat{P}$ of output field $\hat{b}_{\text {out }}$ is detected by $\mathrm{BHD}$, in which the phase change $\delta$ is recorded. The sensitivity of the quantum interferometer is characterized by the uncertainty of a single phase measurement, that is the minimumdetectable phase shift $\Delta \phi$. The calculation details are given in the supplemental material [49-54] and we have the sensitivity 
of interferometer in the lossless case as

$$
\Delta \phi=\sqrt{\frac{\Delta^{2} \hat{P}}{\left(\partial_{\phi} \hat{P}\right)^{2}}}=\sqrt{\frac{(G-g)^{2}}{2\left(I_{p s}-g^{2}\right)}},
$$

where $\partial_{\phi} \hat{P}$ is the change of quadrature phase $\hat{P}$ during the measurement with respect to a phase change $\delta, G$ is the amplitude gain of OPA $\left(|G|^{2}-|g|^{2}=1\right)$ and $I_{p s}$ is the intensity of the phase-sensing light $\left(I_{p s}=\frac{1}{2}(G+g)^{2} I_{0}+g^{2}\right)$. The sensitivity can be enhanced by a factor of $2 G$, when the phasesensing intensity is larger than the square of the gain factor $g^{2}$, which is large enough [49]. The calculation details for the absolute value of the minimum detectable phase $\phi(\Omega)^{\mathrm{min}}$ of the quantum interferometer in the frequency domain are shown in the supplemental material [49, 54]

$$
\phi(\Omega)^{\min }=\sqrt{\frac{4 h c e^{-2 r}}{\lambda G^{\prime} P_{\text {in }}}},
$$

where $h$ is the Plank constant, $c$ is the speed of light in vacuum, $\lambda$ is the laser wavelength, $P_{i n}$ is the input optical intensity of the quantum interferometer, $G^{\prime}$ is the actual power gain factor of the input light, and $r$ is the squeezing parameter associated with shot noise reduction. It is noted that the minimal detectable phase $\phi(\Omega)^{\mathrm{min}}$ is independent of the analysis frequency.

The experimental setup for the OPA-based MZ interferometer is shown in Fig. 1 (b). A Ti:sapphire laser (Coherent MBR-110) with the output power of $2.5 \mathrm{~W}$ pumped by a green laser (Yuguang DPSS FG-VIIIB) is used as the input signal field of the quantum interferometer, fundamental field of second harmonic generation (SHG) and local oscillation field of BHD. The input signal field of the interferometer is splitted on the first linear 50:50 beam splitter $\mathrm{BS}_{1}$ and the two optical beams from $\mathrm{BS}_{1}$ are injected into two OPAs, respectively. The output field from $\mathrm{OPA}_{2}$ is modulated by the sinusoidal signal of $2 \mathrm{MHz}$ through the piezo-electric-transducer $(\mathrm{PZT})_{4}$ to mimic the phase change and then is interfered with the output field from $\mathrm{OPA}_{1}$ on the second linear 50:50 beam splitter $\mathrm{BS}_{2}$. When the signal and noise are measured, the stable bias phase of the interferometer is locked at $\pi+2 k \pi$ ( $k$ is an integer) with the phase locking system based on the PoundDrever-Hall technique and a $\mathrm{PZT}_{3}$ mounted mirror. When the two OPAs are pumped by the vertically polarized $448 \mathrm{~nm}$ continuous-wave single frequency laser from a SHG cavity [49, 55], the OPAs amplify the intensities of phase-sensing lights within the interferometer and squeeze the noises on their phase-quadratures, respectively. The BHD system consisting of a 50:50 beam splitter $\mathrm{BS}_{3}$, two photodiodes and a subtractor. Under the help of the local oscillation light from the laser, the quadrature phase noise power of the output field of interferometer is measured.

The signal and noise levels of the output field of the OPAbased interferometer at the analysis frequency of $2 \mathrm{MHz}$ is shown in Fig. 2. When the optical losses, the mode mismatch at BHD and other imperfection of the interferometer are considered [56], the expression of the interferometric sensitivity

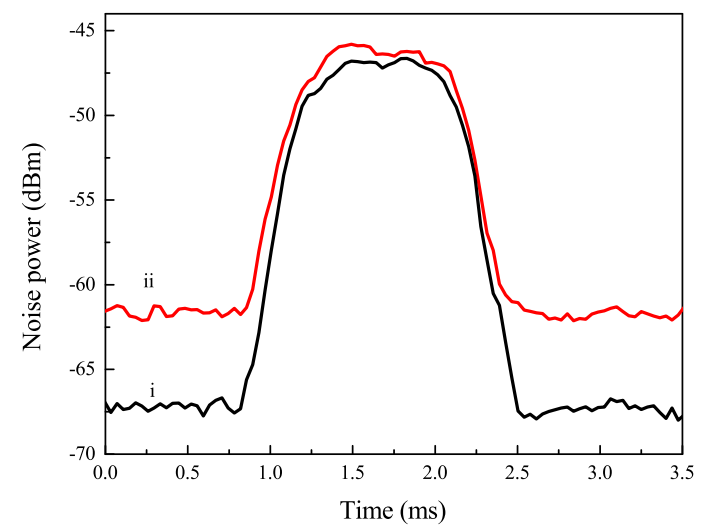

FIG. 2. The signal and noise levels measured at the output field of OPA-based interferometer at analysis frequency of $2.0 \mathrm{MHz}$. The measurement parameters are as follows for the spectrum analyzer : Resolution bandwidth: $100 \mathrm{kHz}$, Video bandwidth: $3 \mathrm{kHz}$, frequency span: 0.

should be dependent on the experimental parameters, which is given by Eq. (12) in the supplemental material [49-51]. The black trace (i) is the output noise power measured at the case of two OPAs operating on the parametric amplification with the OPA gain of 15. The SNL (the red trace (ii)) is measured when the pump fields of two OPAs are blocked and a coherent state as the signal field is injected. The reduction of the shot noise level below the SNL due to squeezing makes the possibility of detecting tiny phase change submerged in the noise ocean. The squeezing of $5.57 \pm 0.19 \mathrm{~dB}$ is the value measured on the output of the OPA-based interferometer when the phase-sensing intensity is amplified from $5 \mu \mathrm{W}$ to $75.3 \mu \mathrm{W}$. When we implement the real measurement the signal light is slightly reduced by the unavoidable loss of $0.71 \mathrm{~dB}$, thus the enhancement of SNR is naturally decreased to $4.86 \pm 0.24 \mathrm{~dB}$ in the comparison with the ideal SNL. Under the same phasesensing intensity of $75.3 \mu \mathrm{W}$, the calculated shot noise spectral densities of quantum interferometer and its corresponding SNL are $6.20 \times 10^{-8} / \sqrt{\mathrm{Hz}}$ and $1.09 \times 10^{-7} / \sqrt{\mathrm{Hz}}$, respectively (according to Eq. (2)).

The effect of the OPA gain $G_{p}$ on the sensitivity of quantum interferometer is shown in Fig. 3 (a), when the input laser power of the interferometer is $10.0 \mu \mathrm{W}$ (the corresponding $\alpha_{i n}^{2}$ of seeded light is $4.5 \times 10^{13} \mathrm{~s}^{-1}$ ). The blue trace (i) corresponds to the SNL; the red trace (ii) and green trace (iii) define the calculated sensitivities of the quantum interferometers when the parameters are taken according to that at actually experimental (see Eq. (12) in the supplementary material) and ideally lossless (see Eq. (1)) conditions, respectively; the purple trace (iv) expresses the quantum Cramér-Rao bound (QCRB) of quantum interferometer (see Eq. (24) in the supplementary material). It can be seen that the sensitivity is improved with the increase of the OPA gain. The observed values of the black squares are worse than the ideal values in trace (iii) because of the influence of losses in the sub- 


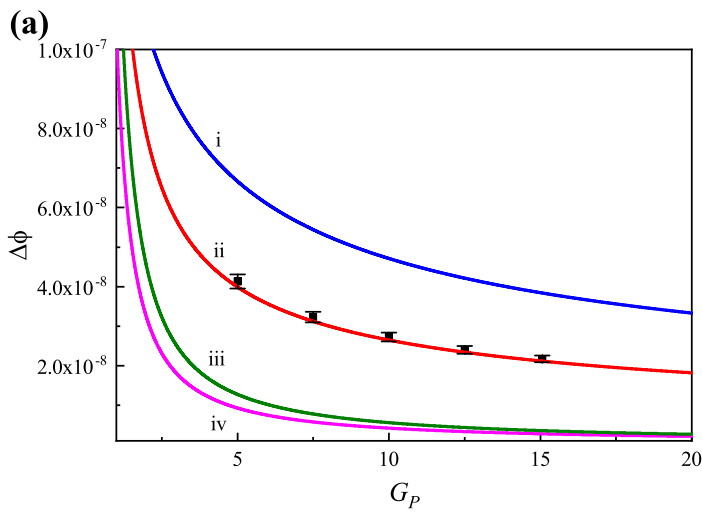

(b)

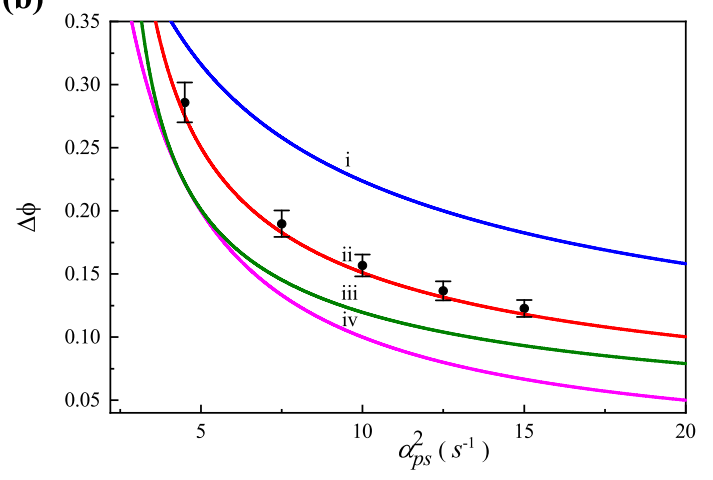

FIG. 3. (a) The effect of the OPA gain $G_{p}$ on the sensitivity of quantum interferometer, when the input laser power of the interferometer is $10.0 \mu \mathrm{W}$. (b) The phase sensitivity of quantum interferometer versus the phase sensing intensity, in which the OPA gain $G_{p}$ of OPA is 5. The black squares and circles indicate the measured sensitivities.

quantum-limit interferometer. When the OPA gain is 15 , the corresponding value of $\Delta \phi_{S N L}$ is $3.8 \times 10^{-8}$ [31]. The calculated value of $\Delta \phi$ can be improved to $3.6 \times 10^{-9}$ in the lossless case, which is 10.6-fold enhancement beyond the above SNL. In the lossless interferometer the corresponding value of $\Delta \phi_{Q C R B}$ is $2.8 \times 10^{-9}$ obtained with the squeezing parameter $\mathrm{r}$ of 1.82 [49, 52, 53]. Therefore, the interferometric phase sensitivity in the lossless case will be close to the QCRB.

The sensitivities of quantum interferometer versus the $\alpha_{p s}^{2}$ of the phase-sensing fields are illustrated in Fig. 3 (b), when the OPA gain $G_{p}$ is 5. The $\alpha_{p s}$ in the horizontal axis stands for the amplitude of the phase-sensing field, which relates to the phase-sensing power $P_{p s}$ by $\alpha_{p s}^{2}=\lambda P_{p s} / h c$. The blue Trace (i) and purple trace (iv) are the SNL and the so-called HL, respectively. The red trace (ii) is the calculated sensitivity of the quantum interferometers in the experimental condition (see Eq. (12) in the supplementary material). The green trace (iii) defines the calculated sensitivity of the quantum interferometers in the improved case when the losses are reduced to $L_{0}=0.002, \eta=0.99$ (see Eq. (12) in the supplementary material). In the improved case with the phase-sensing intensity of $4.5 \mathrm{~s}^{-1}$, the phase sensitivity is 0.22 . The value of the socalled HL calculated with the same intensity phase-sensing fields is also 0.22 [31]. Thus the quantum interferometer is possible to reach the sensitivity allowed by the so-called HL.

In summary, we exploit two OPAs to construct a compact quantum interferometer with a deterministically enhanced phase sensing. The tiny phase change submerged in the SNL can be measured due to both effects of amplified phasesensing intensity and squeezed noise. In the measurement with the low phase-sensing intensity, the phase sensitivity has achieved the Heisenberg-scale precision. The optical losses inside and outside interferometer and the intracavity loss of OPA limit the measurement precision of the present system. The reduction of these losses will enable to obtain better phase-sensing ability. The quantum interferometer is compatible with the SU $(1,1)$ interferometer and squeezed state injection systems, thus they can be combined together for the future sensitivity improvement [57, 58]. Moreover, in the interferometric measurement of the fragile samples, we have to utilize possibly low phase-sensing intensity to protect samples from being damaged. In this case, the squeezed states of light offer a liable option to directly measure tiny signals submerged in the noise ocean. The wavelength used in our interferometer is tunable around $895 \mathrm{~nm}$, which matches not only cesium atom [59] but also biological tissue [60]. Our interferometer is suitable for quantum biology sensing and spectroscopy. Besides the application in the MZ interferometer, the method placing OPAs in interferometer offers a potential to achieve the improvement of sensitivity in other type interferometers with the quantum advantage of the OPA. The presented method promises the unconditional quantum-enhanced precision metrology for any phase related tiny signals.

This research was supported by the National Natural Science Foundation of China (Grants No. 61925503, No. 61775127, No. 11654002, and No. 11834010), the Key Project of the National Key R\&D program of China (Grant No. 2016YFA0301402), the Program for Sanjin Scholars of Shanxi Province, and the fund for Shanxi "1331 Project” Key Subjects Construction.
[1] M. A. Taylor, J. Janousek, V. Daria, J. Knittel, B. Hage, HansA. Bachor, and W. P. Bowen, Phys. Rev. X 4, 011017 (2014).

[2] D. Mason, J. Chen, M. Rossi, Y. Tsaturyan, and A. Schliesser, Nat. Phys. 15, 745(2019).

[3] B. P. Abbott et al. (LIGO Scientific Collaboration and Virgo Collaboration), Phys. Rev. Lett. 116, 061102 (2016).

[4] C. M. Caves, Phys. Rev. D. 23, 1693 (1981).
[5] R. S. Bondurant and J. H. Shapiro, Phys. Rev. D 30, 2548 (1984).

[6] C. L. Degen, F. Reinhard, and P. Cappellaro, Rev. Mod. Phys. 89, 035002 (2017).

[7] Y. Ma, H. Miao, B. H. Pang, M. Evans, C. Zhao, J. Harms, R. Schnabel, and Y. Chen, Nat. Photon. 13, 776 (2017).

[8] B. J. Lawrie, P. D. Lett, A. M. Marino, and R. C. Pooser, ACS 
Photonics 6, 1307 (2019).

[9] M. W. Mitchell, J. S. Lundeen, and A. M. Steinberg, Nature 429, 161 (2004).

[10] V. Giovannetti, S. Lloyd, and L. Maccone, Phys. Rev. Lett. 96, 010401 (2006).

[11] T. Nagata, R. Okamoto, J. L. O’Brien, K. Sasaki, and S. Takeuchi, Science 316726 (2007).

[12] C. Gross, T. Zibold, E. Nicklas, J. Esteve, and M. K. Oberthaler, Nature 464, 1165 (2010).

[13] B. Lucke, M. Scherer, J. Kruse, L. Pezze, F. Deuretzbacher, P. Hyllus, O. Topic, J. Peise, W. Ertmer, J. Arlt, L. Santos, A. Smerzi, and C. Klempt, Science 334, 773 (2011).

[14] M. Xiao, L. A. Wu, and H. J. Kimble, Phys. Rev. Lett. 59, 278 (1987).

[15] P. Grangier, R. E. Slusher, B. Yurke, and A. LaPorta, Phys. Rev. Lett. 59, 2153 (1987).

[16] K. McKenzie, D. A. Shaddock, D. E. McClelland, B. C. Buchler, and P. K. Lam, Phys. Rev. Lett. 88, 231102 (2002).

[17] K. Goda, O. Miyakawa, E. E. Mikhailov, S. Saraf, R. Adhikari, K. McKenzie, R. Ward, S. Vass, A. J. Weinstein, and N. Mavalvala, Nat. Phys. 4, 472 (2008).

[18] T. Eberle, S. Steinlechner, J. Bauchrowitz, V. Handchen, H. Vahlbruch, M. Mehmet, H. Muller-Ebhardt, and R. Schnabel, Phys. Rev. Lett. 104, 251102 (2010).

[19] J. Aasi et al., Nat. Photon. 7, 613 (2013).

[20] M. J. Holland and K. Burnett, Phys. Rev. Lett. 71, 1355 (1993).

[21] S. Slussarenko, M. M. Weston, H. M. Chrzanowski, L. K. Shalm, V. B. Verma, S. W. Nam, and G. J. Pryde, Nat. Photon. 11, 700 (2017).

[22] L. J. Zhang, A. Datta, and I. A. Walmsley, Phys. Rev. Lett. 114, 210801 (2015).

[23] G. Chen, L. J. Zhang, W. H. Zhang, X. X. Peng, L. Xu, Z. D. Liu, X. Y. Xu, J. S. Tang, Y. N. Sun, D. Y. He, J. S. Xu, Z. Q. Zhou, C. F. Li, and G. C. Guo, Phys. Rev. Lett. 121, 060506 (2018).

[24] K. C. McCormick, J. Keller, S. C. Burd, D. J. Wineland, A. C. Wilson, and D. Leibfried, Nature 572, 86 (2019).

[25] W. Wang, Y. Wu, Y. Ma, W. Cai, L. Hu, X. Mu, Y. Xu, Z. Jie, H. Wang, Y. P. Song, H. Yuan, C. L. Zou, L. M. Duan, and L. Sun, Nat. Commun. 281, 4382 (2019).

[26] Z. Hou, R. J. Wang, J. F. Tang, H. D. Yuan, G. Y. Xiang, C. F. Li, and G. C. Guo, Phys. Rev. Lett. 123, 040501 (2019).

[27] L. Pezze and A. Smerzi, Phys. Rev. Lett. 100, 073601 (2008).

[28] P. M. Anisimov, G. M. Raterman, A. Chiruvelli, W. N. Plick, S. D. Huver, H. Lee, and J. P. Dowling, Phys. Rev. Lett. 104, 103602 (2010).

[29] B. Yurke, S. L. McCall, and J. R. Klauder, Phys. Rev. A 33, 4033 (1986).

[30] W. N. Plick, J. P. Dowling, and G. S. Agarwal, New J. Phys. 12, 083014 (2010).

[31] Z. Y. Ou, Phys. Rev. A 85, 023815 (2012).

[32] F. Hudelist, J. Kong, C. J. Liu, J. T. Jing, Z. Y. Ou, and W. P. Zhang, Nat. Commun. 5, 3049 (2014).
[33] M. Manceau, G. Leuchs, F. Khalili, and M. Chekhova, Phys. Rev. Lett. 119, 223604 (2017).

[34] B. E. Anderson, P. Gupta, B. L. Schmittberger, T. Horrom, C. H. Avigliano, K. M. Jones, and P. D. Lett, Optica 4, 752 (2017).

[35] P. Gupta, B. L. Schmittberger, B. E. Anderson, K. M. Jones, and P. D. Lett, Opt. Exp. 26, 391 (2018).

[36] P. N. Prajapati and I. Novikova, Opt. Lett. 44, 5921(2019).

[37] R. C. Pooser and B. Lawrie, Optica 2, 393 (2015).

[38] R. C. Pooser et al. arXiv:1912.10550

[39] C. Vitelli, N. Spagnolo, L. Toffoli, F. Sciarrino, and F. DeMartini, Phys. Rev. Lett. 105, 113602 (2010).

[40] A. S. Coelho, F. A. S. Barbosa, K. N. Cassemiro, A. S. Villar, M. Martinelli, and P. Nussenzveig, Science 326, 823 (2009).

[41] J. Roslund, R. Medeiros de Araujo, S. F. Jiang, C. Fabre, and N. Treps, Nat. Photonics 8, 109 (2014).

[42] M. Chen, N. C. Menicucci, and O. Pfister, Phys. Rev. Lett. 112, 120505 (2014).

[43] Z. H. Yan, L. Wu, X. J. Jia, Y. H. Liu, R. J. Deng, S. J. Li, H. Wang, C. D. Xie, and K. C. Peng, Nat. Commun. 8, 718 (2017).

[44] Y. Y. Zhou, J. Yu, Z. H. Yan, X. J. Jia, J. Zhang, C. D. Xie, and K. C. Peng, Phys. Rev. Lett. 121, 150502 (2018).

[45] M. R. Huo, J. L. Qin, J. L. Cheng, Z. H. Yan, Z. Z. Qin, X. L. Su, X. J. Jia, C. D. Xie, and K. C. Peng, Sci. Adv. 4, 9401 (2018).

[46] W. Asavanant, Y. Shiozawa, S. Yokoyama, B. Charoensombutamon, H. Emura, R. N. Alexander, S. Takeda, J. Yoshikawa, N. C. Menicucci, H. Yonezawa, and A. Furusawa, Science 366, 373 (2019).

[47] M. V. Larsen, X. Guo, C. R. Breum, J. S. N.-Nielsen, and U. L. Andersen, Science 366, 369 (2019).

[48] H. Vahlbruch, M. Mehmet, K. Danzmann, and R. Schnabel, Phys. Rev. Lett. 117, 110801 (2016).

[49] X. J. Zuo, Z. H. Yan, Y. N. Feng, J. X. Ma, X. J. Jia, C. D. Xie, and K. C. Peng, Phys. Rev. Lett. 124, 173602 (2020).

[50] H. A. Bachor and T. C. Ralph, A Guide to Experiments in Quantum Optics (Wiley-vch, Weinheim, Berlin, 2004).

[51] T. Tanimura et al. Opt. Lett. 31, 2344 (2006).

[52] D. Li, B. T. Gard, Y. Gao, C. H. Yuan, W. P. Zhang, H. Lee, and J. P. Dowling, Phys. Rev. A 94, 063840 (2016).

[53] C. Sparaciari, S. Olivares, and M. G. A. Paris, Phys. Rev. A 93, 023810 (2016).

[54] K. Goda, Development of Techniques for Quantum-Enhanced Laser-Interferometric Gravitational-Wave Detectors Thesis, Massachusetts Institute of Technology (2007).

[55] X. J. Zuo, Z. H. Yan, and X. J. Jia, Appl. Phys. Express 12, 032010 (2019).

[56] Y. Eto, T. Tajima, Y. Zhang, and T. Hirano, Opt. Lett. 32, 1698 (2007).

[57] M. Tse et al. Phys. Rev. Lett. 123, 231107 (2019).

[58] F. Acernese et al. (Virgo Collaboration) Phys. Rev. Lett. 123, 231108 (2019).

[59] R. Ma, W. Liu, Z. Z. Qin, X. J. Jia, and J. R. Gao, Phys. Rev. A 96, 043843 (2017).

[60] J. L. Sandell and T. C. Zhu, J. Biophotonics. 4, 773 (2011). 